\title{
Emphasizing expert practice with spaced recall
}

\author{
Eugene Torigoe, and Dominic Licata \\ Department of Physics, Thiel College, Greenville, PA 16125
}

\begin{abstract}
In this paper we discuss a methodology incorporating spaced recall to emphasize long-term retrieval of worked example problems. We believe that the structure of this activity will help students understand the utility of expert practices such as translating between representations, and reasoning through a problem rather than just memorizing every detail. We collected data from 4 interviews, and from the written work of 11 students in introductory physics. Most students found the exercise useful, but we believe that it could be improved by improving the quality of the feedback.
\end{abstract}

\section{INTRODUCTION}

The term "memorization without understanding" is a common description in the PER literature for the types of poor and unproductive strategies that many students use in introductory physics. $[1,2]$ In contrast, an expert's understanding of physics is described as entailing "conceptual understanding", "using and understanding multiple representations", "drawing diagrams". [3-6]

We often encounter students whose primary strategy for learning physics can best be described as "memorization without understanding". We observe students who memorize the details of example problems, but who can't describe the reasoning behind the steps in the solution, and are flummoxed by variations of those problems on exams. This paper is the outcome of our goal to help students transition from strategies like "memorization without understanding" toward expert-like strategies.

The PER community has developed many inventive activities that promote expert practices. [7] One general approach is to create tasks that break the traditional problem solving structure, and focus on, for example, conceptual reasoning. These activities change the reward structure to encourage reasoning rather than memorization. For example, TIPERS [8], conceptual tutorials [9], and Invention tasks [10].

We propose a new method that relies on the spaced recall of information. Memory is often categorized as either being stored in the short-term or long-term memory. The shortterm memory is very volatile, so while it is retrievable in the short-term, it is easily forgotten. The long-term memory is much more stable, and reliable over long time periods. Research on human memory has demonstrated that one of the most effective methods of storing information in one's longterm memory is to periodically recall it over time. Essentially this method relies on the interrogation of one's own long-term memory. [11] This spaced recall methodology been shown to be more effective than re-reading, or re-presentation. [12]

The basic structure of the activity as we have implemented is detailed below:

1. View a video of a worked example problem, while taking notes.

2. Study notes, and then recall the solution without assistance only a few minutes after watching the video (immediate recall). Then use notes from the video for feedback as necessary. The notes are collected by the instructor.

3. A week later, recall the solution (spaced recall). And repeat this spaced recall once a week, over a period of weeks. Use notes from the video for feedback after each recall as necessary.

\section{Solve a related transfer problem.}

This is different from other PER interventions because it does not make the "memorization without understanding" strategy untenable. But, it is designed to make such a strategy cumbersome. When the goal is long-term recall, memorization of disconnected facts is very difficult. A more productive method, which we believe characterizes the expert approach, is to strategically memorize information and then reason through the rest of the solution. When experts remember a derivation, they do not remember all of the details of the derivation, but instead strategically remember general equations, general rules, and pictures to figure out the final result. Experts reconstruct the majority of the derivation. Richard Feynman referred to this skill as "triangulation".

There comes a time —which you haven't quite got to, yet —where you'll know so many things that as you forget them, you can reconstruct them from the pieces that you can still remember. It is therefore of first-rate importance that you know how to "triangulate" — that is, to know how to figure something out from what you already know. It is absolutely necessary. [13]

We believe that these types of long-term retrieval tasks will serve as a demonstration to the students of the value to general procedures like the translation between representations, and starting from general principles. And so an expert-like approach to recalling this problem was emphasized when the solution was first presented.

In order to increase student buy-in, and to encourage the expert approach we structured this activity as a formative assessment. Students where given points for participating, but not on their performance.

When we were formulating this activity we choose problems that would highlight the following expert skills: 
1. Using diagrams to generate equations

2. Using conceptual ideas to organize problem solving

3. Solving problems symbolically

\section{Starting from general equations}

This approach however, does not encompass all aspects of the expert problem solving. For example, the exercise we describe here does not require students to plan a strategy, check their results, distinguish relevant from irrelevant information, or to choose between multiple possible solution paths.

\section{DATA COLLECTION}

We used two methods to collect data. Speak aloud interviews were performed with 4 former physics students as they went through the procedure. Students did all of their written work on a white board, and were asked to describe their reasoning to an interviewer.

In addition, 11 calculus-based introductory physics students were assigned this procedure during their lab period. We collected their written work, and at the end of the activity we gave them a survey which asked them to reflect on the activity. We developed three problems for this activity: Heat engine efficiency problem, pendulum in accelerating car problem, and conserving momentum during a collision problem.

Most of the students in the study were either Sophomores or Juniors, and were either a math or a non-physics science major. Only one student in the study was a physics major.

\section{Heat Engine:}

A heat engine operates between a hot thermal reservoir at a temperature $T_{H}$ and a cold thermal reservoir at $T_{C}$. Create an energy flow diagram for the thermal reservoirs and the heat engine, and use it to derive a symbolic expression for the thermal efficiency, $\eta$, (pronounced eta) of the heat engine in terms of the heat from the high temperature reservoir $Q_{H}$ and the heat from the cold temperature reservoir $Q_{C}$.

\section{Collision:}

Two balls traveling along the $\mathrm{x}$-axis collide. Ball $\mathrm{A}$ has a mass of $\mathrm{m}$, and a velocity of $11 \mathrm{v}$ to the right. Ball $\mathrm{B}$ has a mass of $2 \mathrm{~m}$, and a velocity of $\mathrm{v}$ to the left. When the balls collide, they stick together. Use the conservation of momentum to determine the speed and direction of the two balls after the collision in terms of $\mathrm{m}$ and/or $\mathrm{v}$.

Momentum: $p=m v$

Conservation of momentum: $p_{A i}+p_{B i}=p_{A f}+p_{B f}$

\section{Pendulum:}

A pendulum (a ball connected to a thin string) has a mass $m$ is hung from the rear view mirror of a car. When the car is at rest the pendulum hangs down in the vertical direction. When the car has constant acceleration in the forward direction the pendulum is at a stable angle $\theta$ from the vertical. Draw a free body diagram for the forces on the mass $m$ due to the gravitational force $\mathrm{mg}$ and the tension T. Use Newton's 2nd Law to find an expression for the acceleration, a, in terms of the angle $\theta$ and the gravitational constant $\mathrm{g}$.

Newton's 2nd Law in the s-direction: $\Sigma F_{s}=m a_{s}$

Trigonometry: $\sin \theta=$ opp/hyp, $\cos \theta=a d j / h y p$, $\tan \theta=o p p / a d j$

In addition, we wrote transfer problems for each of the problems which we designed to have similar steps, but with slight variations to see if students could apply the same reasoning to a new situation.

For the heat engine alternate problem, a diagram was provided to the student that showed the diagram from the original problem with a heat flow labeled $Q_{H 1}$ from the high temperature reservoir to the heat engine, but with an additional heat flow from the high temperature reservoir to the low temperature reservoir labeled $Q_{H 2}$. This problem was designed to see if the students could properly reason when to use $Q_{H 1}$, and $Q_{H_{2}}$ in their equations.

For the collision alternate problem, a object has an initial unknown velocity, and then it explodes into two pieces, with given masses and velocities. This problem was designed to see if the students could use the same methodology to setup the conservation of momentum problem to solve for a different unknown.

For the pendulum alternate problem, a diagram was provided showing the car accelerating at a constant rate down an incline, a diagram showing the pendulum, and a diagram of the free body diagram with rotated axes that are parallel and perpendicular to the incline. This problem is different from the original because one has to take components of the weight, but not the tension.

\section{RESULTS}

In both the interviews and in the course a very similar pattern was observed (see Fig. 1).

1. Students do very well at recall immediately after having watched the video solution.

2. Student recall performance drops after a 1 week break.

3. Student recall performance tends to either remain steady or improve after more spaced recall attempts.

Two of the students in the class seemed to give up on the exercises after a few weeks. They did not get much feedback, and perhaps their written work was not enough feedback to keep them engaged.

There were a handful of students who did not make any mistakes on any of the exercises, although many of this group of students remarked in the survey that they changed their approach from memorization to reasoning through the problem. 


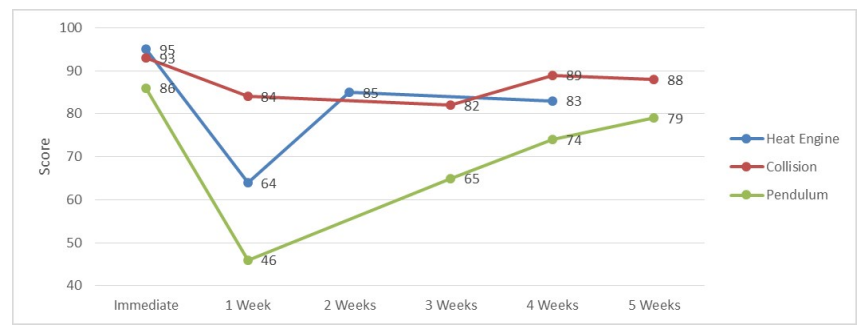

FIG. 1. Score for the spaced recall problems for 11 students. Gaps exist because not every problem being give each week.

\section{A. Surveys}

Almost all of the students described in the survey that they thought the exercises were helpful. Many expressed that while at first they tried to just memorize the solutions, they later found that reasoning through the problem was the superior method. Below is a sample survey response.

Yes, by repeating the same problem over and over it helped me to truly learn the setup of equations and why they work the way they do.

About a third of the students mentioned that they made a transition from just trying to memorize the solution to reasoning through them.

At first I just memorized it. However, as time progressed I approached the problems with more reason

At first memorized, but when began to reason through (a lot easier)

One student specifically mentioned their difficulty on the transfer problem as being a motivating factor to not just memorize the solution.

The [transfer problems] helped because I was able to apply basic/general techniques from the original ones to the new q's. The first question we did [heat engine problem] I feel like I just memorized the problem and had trouble applying the techniques [to the transfer problem].

Of course, not all students changed their problem solving methodology. For example, one student wrote:

I tried to memorize rather than reason through it.

\section{B. Transfer Problems}

Performance on the alternate transfer problems was very variable. For the heat engine transfer problem, students were

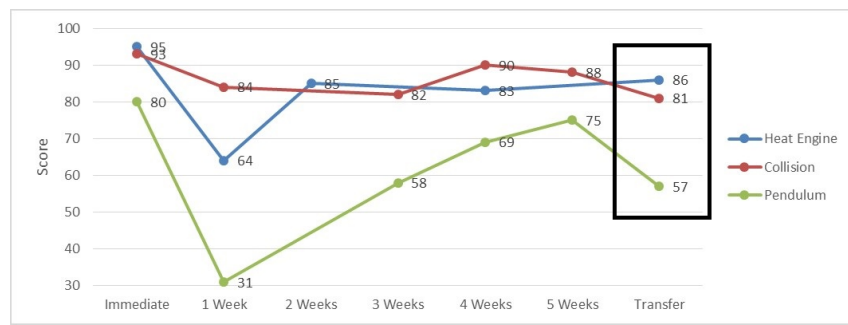

FIG. 2. Score for the recall problems as well as the transfer problem (highlighted by the black rectangle) for 9 students who completed all recall problems as well as the transfer problem. This data includes the score for components that are common for the original and transfer problems.

in general able to apply the same techniques to the new problem, but for the other transfer problems, the performance of the students dropped. It appears that the rotation of the axes in the pendulum transfer problem made the problem much more difficult than the original problem. Figure 2 was created to include only the students who completed all of the problems, and only includes scores for parts of the problems that appear in both the original and the transfer problems. For example, if the transfer problem included a diagram, drawing the diagram for the original problem was not scored.

\section{Interviews}

During the interviews the same pattern was observed. The subjects were very successful recalling the solutions within minutes of having watched the video of the solution (immediate recall). When questioned about their procedure, they were also able to explain the reasoning for each of the steps. During the first spaced recall a week or two later, all of the students made errors in their solutions. Some were completely lost, while others only made minor errors. During subsequent spaced recall sessions, the subjects improved, and by the end of the interviews all of the subjects were able to solve the problems correctly with only minor errors.

During the last interview the subjects were asked about what they thought about the procedure. The two subjects who struggled the most during the first spaced recall session remarked that the activity changed the way they thought about problem solving. These two students received feedback that emphasized using the diagram to generate the equations.

So tell me, do you think there is any change in the way you use diagrams since when you started this process?

Yeah a little more I learned to look into the actual diagram, and look at what it is telling me, not that it's just there as a picture to help explain the problem

The student had treated the diagram as a representation of 
the problem to be solved instead of as a tool that could aid in the problem solving process.

You know I'll tell you that one of the things I noticed, when we did the first two interviews I felt like you weren't kind of using the pictures as much as you could have...

Yeah I remember how it went through. I never really looked through diagrams as a tool, I just looked at them as they were just there, that's what it looks like, I never plugged things in and solved an equation with it ... somehow it taught me how to actually use the diagram, that's one of the main things that I'll pull away from this, if I have a class in the future that uses diagrams

The other two interview subjects made only minor errors during the spaced recall sessions, and received feedback on their errors. These subjects felt that these exercises helped them remember the solution, but did not mention that it had any influence on how they approached problem solving.

One of these two interview subject described how her difficulty with the transfer problem demonstrated that she did not understand the reasoning for all of the steps of the original problem. This demonstrated to us that the transfer problems could be used as a way to also give the students a gauge as to how well they understood the reasoning behind each of the steps.

OK, so with the pendulum [transfer] problem. You don't think that the previous weeks helped you to at least

No I think for the most part, I think I just memorized how that problem worked in that spe- cific example, and I didn't actually fully understand what would do in like other situations with pendulum, and what you would do if you were not on a flat surface.

\section{CONCLUSIONS}

This work stems from our goal to help students transition from novice to more expert-like strategies. For many of the students this was an effective procedure to get them to understand the benefit of using expert procedures. In particular, some students saw the benefit of using the diagrams to generate equations, and to differentiate memorization from derivation.

One source of difficulty with this procedure is that it is very difficult to distinguish in the students' written work, which students are using expert practices from just memorizing without understanding. We believe that the structure as a formative activity is an important factor in getting students to try a non-memorized approach. More work is needed to determine if the methodology described in this paper is the optimum method to get students to transition from memorizing to reasoning. A weakness of this work is our low students numbers. If you would like to contribute data from your students, then please contact us.

We plan on continuing this procedure next year. To improve the exercise we are planning to include presentations by the instructor after each recall to improve the quality of the feedback. More transfer problems to show them the benefit of understanding the reasoning behind each step. Better tracking of students to intervene before students give up. Pre and post course survey data on student epistemologies. And opportunities for the students to reflect on their problem solving, and how it has evolved while in the class.
[1] Elby, Andrew. "Helping physics students learn how to learn." American Journal of Physics 69.S1 (2001): S54-S64.

[2] Hammer, David. "Epistemological beliefs in introductory physics." Cognition and Instruction 12.2 (1994): 151-183.

[3] Van Heuvelen, Alan. "Learning to think like a physicist: A review of research-based instructional strategies." American Journal of Physics 59.10 (1991): 891-897.

[4] Chi, M. T. H., Feltovich, P. J. and Glaser, R. (1981), Categorization and Representation of Physics Problems by Experts and Novices. Cognitive Science, 5: 121-152.

[5] Larkin, J., McDermott, J., Simon, D. P., and Simon, H. A. (1980). Expert and novice performance in solving physics problems. Science, 208, 1335-1342.

[6] Kohl, Patrick B., and Finkelstein, Noah D. "Patterns of multiple representation use by experts and novices during physics problem solving.” Phys. Rev. ST Phys. Educ. Res. 4, 010111 Published 9 June 2008

[7] Hsu, Leonardo, et al. "Resource letter RPS-1: Research in problem solving." American Journal of Physics 72.9 (2004): 1147-1156.

[8] Hieggelke, C. J., et al. "Tipers Sensemaking Tasks for Introductory Physics.” (2014).

[9] McDermott, Lillian C., and Peter S. Shaffer. Tutorials in introductory physics. Prentice Hall, 1998.

[10] Brahmia, Suzanne White, Mathematization in introductory physics. Diss. Rutgers University, 2014

[11] Roediger, Henry L., and Jeffrey D. Karpicke. "The power of testing memory: Basic research and implications for educational practice." Perspectives on Psychological Science 1.3 (2006): 181-210.

[12] Brown, Peter C., Roedinger, Henry L., and McDaniel, Mark A. Make it Stick the Science of Successful Learning. Cambridge: Belknap Press, 2014. Print.

[13] Feynman, Richard Phillips, et al. Feynman's tips on physics. Pearson, 2006. 